\title{
BMJ Open Validation of intracardiac shunt using thoracic bioimpedance and inert gas rebreathing in adults before and after percutaneous closure of atrial septal defect in a cardiology research unit: study protocol
}

\author{
Laura Filaire, ${ }^{1}$ Aurelie Chalard, ${ }^{2}$ Hélène Perrault, ${ }^{3,4}$ Romain Trésorier, ${ }^{2}$ \\ Jean-René Lusson, ${ }^{2}$ Bruno Pereira, ${ }^{5}$ Frederic Costes, ${ }^{6,7}$ Claire Dauphin, ${ }^{2}$ \\ Ruddy Richard ${ }^{4,6,7}$
}

To cite: Filaire $L$, Chalard $A$, Perrault $\mathrm{H}$, et al. Validation of intracardiac shunt using thoracic bioimpedance and inert gas rebreathing in adults before and after percutaneous closure of atrial septal defect in a cardiology research unit: study protocol. BMJ Open 2019;9:e024389. doi:10.1136/ bmjopen-2018-024389

- Prepublication history for this paper is available online. To view these files, please visit the journal online (http://dx.doi org/10.1136/bmjopen-2018024389).

Received 25 May 2018 Revised 6 March 2019 Accepted 21 March 2019
Check for updates

(C) Author(s) (or their employer(s)) 2019. Re-use permitted under CC BY-NC. No commercial re-use. See rights and permissions. Published by BMJ.

For numbered affiliations see end of article.

Correspondence to Professor Ruddy Richard; rrichard@chu-clermontferrand.fr

\section{ABSTRACT}

Introduction Intrathoracic shunt quantification is a major factor for appropriate clinical management of heart and pulmonary diseases. Intracardiac shunts quantified by pulmonary to systemic output ratio (Qp/ Qs) are generally assessed by Doppler echocardiography, MRI or catheterisation. Recently, some authors have suggested the concomitant use of thoracic bioimpedance (TB) and inert gas rebreathing (IGR) techniques for shunt quantification. The purpose of this study is to validate the use of this approach under conditions where shunt fraction is directly quantified such as in patients with isolated atrial septal defect (ASD).

Methods and analysis This trial is a prospective, observational single-centre, non-blinded study of adults seen for percutaneous closure of ASD. Qp/Qs ratio will be directly measured by Doppler echocardiography and direct Fick. IGR and TB will be used simultaneously to measure the cardiac output before and after closure: the ratio of outputs measured by IGR and TB reflecting the shunt fraction. The primary outcome will be the comparison of shunt values measured by TB-IGR and Doppler echocardiography.

Ethics and dissemination The study has been approved by an independent Research Ethics Committee (2017A03149-44 Fr) and registered as an official clinical trial. The results will be published in a peer-reviewed journal. Trial registration number NCT03437148; Pre-results.

\section{INTRODUCTION}

Intrathoracic shunt quantification is a major parameter for the clinical management of cardiac and pulmonary diseases. Several techniques are currently available for quantification but present some limitations: the direct Fick method is invasive and therefore not first indication, easy access to MRI remains an issue in several clinical centres while the

\section{Strengths and limitations of this study}

- The validation of a double non-invasive approach for assessing the extent of cardiac shunt will provide an alternative to catheterisation.

- The double non-invasive approach is used concomitantly to an arteriovenous catheterisation.

- The advantage of the bioimpedance method for stroke volume determination is that it is continuous and does not require patient participation.

- A limitation of inert gas rebreathing resides in the lack of control on patient rebreathing manoeuvres.

precision and repeatability of Doppler echocardiography measurements is highly dependent on technical ability and experience.

Recently, Perrault et al suggested a new approach of intrathoracic shunt quantification by using concomitantly thoracic bioimpedance (TB) and inert gas rebreathing (IGR). TB allows the determination of the systemic cardiac output (Qs) and IGR enables the assessment of the pulmonary blood flow

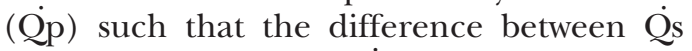
measured by TB and Qp determined from the IGR measurement may be taken to reflect the intrathoracic shunt. The extent to which the use of these combined non-invasive techniques provides quantification that is sensitive enough to be of use in the clinical management of patients with intrathoracic shunts remains to be validated.

Isolated atrial septal defect (ASD) is a congenital heart disease leading to intracardiac left-to-right shunts of varying degrees depending on size of defect and left ventricular compliance. ${ }^{2}$ Clinical management 
generally entails assessment of $\dot{\mathrm{Qp}} / \dot{\mathrm{Qs}}$ ratio since the clinical decision for percutaneous closure is highly dependent on the magnitude of the left-to-right shunt. ${ }^{34}$

The comparison of shunt quantification using the combined TB and IGR techniques with that obtained through routine measurements in isolated ASD provides an opportunity to validate the usefulness of this approach in clinical settings. The aim of this study is thus to validate the combined non-invasive approach of $\dot{Q p} / \dot{\mathrm{Q} s}$ ratio measurement using TB and IGR in adults with secundum ASD by comparing results obtained with those from usual methods of Doppler echocardiography and direct Fick.

\section{METHODS AND ANALYSIS \\ Setting}

The present trial is a prospective, single-centre, observational, non-blinded study that compares the measurement of the pulmonary to systemic blood flow ratio (Qp/Qss) by the concomitant use of IGR and TB first to the non-invasive Doppler echocardiography before and after defect closure and, second, to the direct Fick method during the defect closure procedure.

\section{Study objectives}

The aim of the study is to validate the combined non-invasive $\dot{\mathrm{Qp}} / \dot{\mathrm{Q} s}$ ratio measurement in adults with ASD both before and after interventional closure in comparison to the Doppler echocardiography measure and during the closure procedure to the direct Fick method.

\section{Methods}

Methodology and design

The study will take place in the cardiology unit of the University Hospital Center, with the view to include 30 patients over a period of 2 years from inclusion of first patient. Patients will be enrolled and followed by cardiologists who will perform right heart catheterisation and Doppler echocardiography. The non-invasive measurement of QpigR / Qs $_{\mathrm{TB}}$ using IGR and TB will be made before and after closure by trained clinical investigators without knowing the results obtained by cardiologists at the time of data acquisition.

\section{Patients and public involvement}

This research was designed by clinicians with the aim of simplifying patient's management. Patients were not involved in the recruitment to or conduct of the study. According to the law in force in France, participants will be individually informed of the results at the end of the study.

\section{Inclusion criteria}

Patients with the following conditions will be included:

- Adult patients ( $\geq 18$ years old) with confirmed secundum ASD with indication for interventional closure. $^{5}$
- ASD regardless of the size, with suspicion of paradoxical embolism.

- Patients with significant intracardiac shunt (right ventricular volume overload) and pulmonary vascular resistance (PVR) $<5$ Wood units (WU).

- Patients with PVR $\geq 5$ WU but $<2 / 3$ systemic vascular resistance or pulmonary arterial pressure $<2 / 3$ systemic pressure and evidence of net left-to-right shunt (Qp/Qs $>1.5)$.

\section{Exclusion criteria}

Patients will be excluded for the study if they present with one of the following criteria:

- Pregnant and breastfeeding women.

- Patient under assisted ventilation.

- Chronic respiratory disease ventilation/perfusion mismatch abnormalities.

- Complex congenital heart disease.

- Patients with coagulation function abnormalities.

- Patients who have not provided written consent.

- Patients in whom the use of TB or IGR is contraindicated.

\section{ASD closure}

Patients with secundum ASD eligible for percutaneous closure will be invited to participate in the study and to provide their signed informed consent. The percutaneous closure will be performed during a 3-day hospitalisation as per the usual institutional protocol. Resting haemodynamic measurements of shunt flow $\left(\dot{Q p} / \mathrm{Qs}_{\mathrm{ED}}\right.$ ), systemic cardiac output ( $\dot{\mathrm{Q} s})$ and right and left ventricular functions will be performed using Doppler echocardiography the day before as well as the day after percutaneous closure. Concomitantly, pulmonary blood flow by IGR (QpigR) and the systemic cardiac output by TB $\left(\mathrm{Qs}_{\mathrm{TB}}\right)$ will be measured. Right heart catheterisation will also be performed to directly measure $\dot{Q p}$ and Qs through a direct Fick computation ( $\left.\dot{\mathrm{Qp}} / \mathrm{Qs}_{\text {Fick }}\right)$. An 'Amplatzer Septal Occluder (Saint Jude)' will be used for ASD closure.

The sequence of procedures is shown in figure 1.

\section{Study group}

In this study, subjects serve as their own control for all relevant measures.

\section{Approaches for cardiac output determination and shunt fraction quantification \\ Doppler echocardiography}

Cardiac output measurement will be obtained using standard cardiac ultrasound (Ge-Vid S9, Philips-IE 33 and Philips-EPIC). Images will be captured in parasternal (short and long axes) and apical four-chamber views with 2D cardiac ultrasound with patient in left lateral decubitus. Cross-sectional area of the pulmonary annulus (Ap) and aortic annulus (Aa) will be measured. ${ }^{6}$ Velocity of blood will be assessed by pulsed Doppler. Velocity at the pulmonary annulus and aortic annulus is plotted on time to obtain the pulmonary and systemic velocity time 


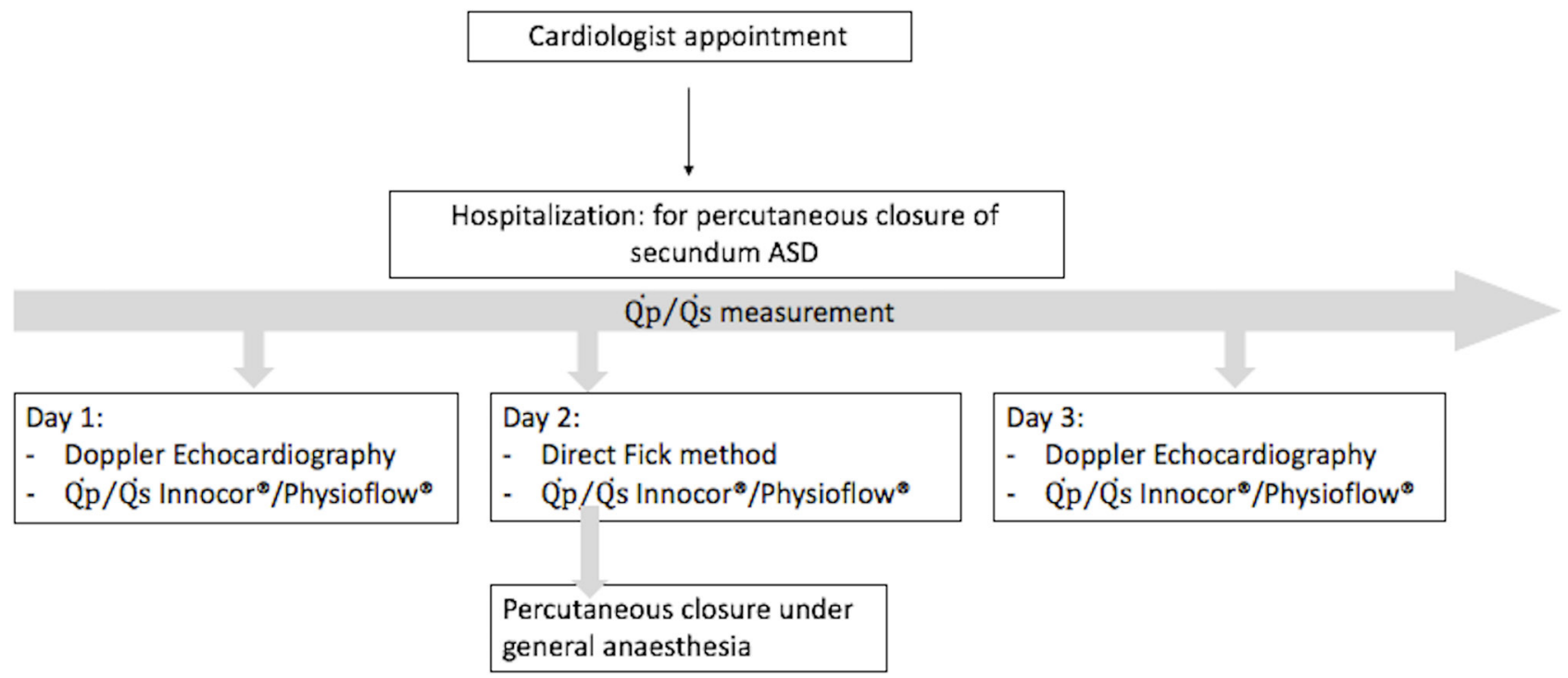

Figure 1 Consort diagram: flow chart. Patients indicated to ASD percutaneous closure will be proposed to the study. Three assessments of the ratio with the concomitant use of IGR and TB are planned during a 3-days hospitalization: the day before and after intervention in comparison to the Doppler Cardiac Ultrasound measure and one the day of intervention in comparison to the direct Fick method. ASD: atrial septal defect.

integral (VTIp and VTIa, respectively). Pulmonary and systemic cardiac output measurements are based on the following formulae. ${ }^{7}$

$$
\begin{aligned}
& \dot{\mathrm{Qp}}\left(\mathrm{mL} \cdot \mathrm{min}^{-1}\right)=\mathrm{HR}\left(\text { beats } \cdot \mathrm{min}^{-1}\right) \times \operatorname{Ap}\left(\mathrm{cm}^{2}\right) \times \operatorname{VTIp}(\mathrm{cm}) \\
& \dot{\mathrm{Q}}\left(\mathrm{mL} \cdot \mathrm{min}^{-1}\right)=\mathrm{HR}\left(\text { beats } \cdot \mathrm{min}^{-1}\right) \times \operatorname{Aa}\left(\mathrm{cm}^{2}\right) \times \operatorname{VTIa}(\mathrm{cm})
\end{aligned}
$$

The echocardiographic shunt fraction is estimated from $\dot{Q}$ p/Qs ratio with values from three non-continuous cardiac cycles being used for computation. In turn, the computed values will serve as reference for statistical analyses. ${ }^{8}$

\section{Fick quantification of shunt}

Under local anaesthesia and after pressure and ECG monitoring, a catheter will be inserted through the right femoral vein allowing blood gas samples at various sites and then pushed through the ASD for pulmonary vein sampling to enable oxygen saturation comparisons and left-to-right shunt contribution.

The standard Fick equation will be applied for computation of cardiac output to include or exclude shunted blood fractions.

Systemic cardiac output will be taken as:

$$
\text { QS Fick }\left(\mathrm{mL} \cdot \min ^{-1}\right)=\dot{\mathrm{VO}}_{2}\left(\mathrm{~mL} \cdot \min ^{-1}\right) /\left[\mathrm{C}_{\mathrm{a}} \mathrm{O}_{2}-\mathrm{C}_{\overline{\mathrm{v}}} \mathrm{O}_{2}\left(\mathrm{~mL} \cdot \min ^{-1}\right)\right] \text { where }
$$

$\dot{\mathrm{V}} \mathrm{O}_{2}=$ whole body oxygen consumption.

$\mathrm{C}_{2} \mathrm{O}_{2}=$ arterial oxygen content in the femoral artery.

$\mathrm{C}_{-} \mathrm{O}_{2}=$ mixed venous oxygen content obtained from the oxygen saturation and haemoglobin concentration in inferior vena cava (IVC) and superior vena cava (SVC) especially for patients with ASD. ${ }^{9}$

Shunt contribution will be obtained through a series of Fick computations using successive venous content values of samples in the IVC, the SVC, the innominate vein, the right atrium and ventricle and the pulmonary artery.
The oxygen content measured at each of these sites will be introduced into the Fick equation to determine the corresponding cardiac output, reflecting the absence or presence of blood shunt contribution.

\section{Double non-invasive cardiac output measurement}

This approach will be based on the use of two cardiac output determination methods simultaneously, leading to intrathoracic shunt quantification: TB measurement reflects Qs while IGR reflects Qp (figure 2).

\section{Thoracic bioimpedance}

The methodology of PhysioFlow (Manatec, France) has been well described in the study of Charloux et al. ${ }^{10}$ TB was validated against the Fick method in healthy subjects ${ }^{11}$ and in patients with chronic heart and respiratory diseases. ${ }^{1012-14}$ Two sets of cutaneous electrodes are placed on the thorax of the subject to reflect changes in bioimpedance. The changes in thoracic impedance $(\Delta \mathrm{Z})$ occurring as a result of cardiac ejection are continuously recorded and reflect the stroke volume (SV). Systemic cardiac output is then determined from the multiplication of SV and heart rate. The Qs the average of continuous data over the last $30 \mathrm{~s}$ (approximately 25-30 heartbeats) immediately prior to the rebreathing manoeuvres.

\section{Inert gas rebreathing}

IGR is validated against invasive method Fick and thermodilution for cardiac output measurement in both healthy subjects and patients with heart disease. ${ }^{15-18}$ The Innocor (Innovision, Odense, Denmark) device, which will be used in this study, measures the QpigR using the rebreathing technique with a mixture of gases (sulfur hexafluoride $\left(\mathrm{SF}_{6}\right)$, nitrous oxide $\left(\mathrm{N}_{2} \mathrm{O}\right)$ and oxygen 


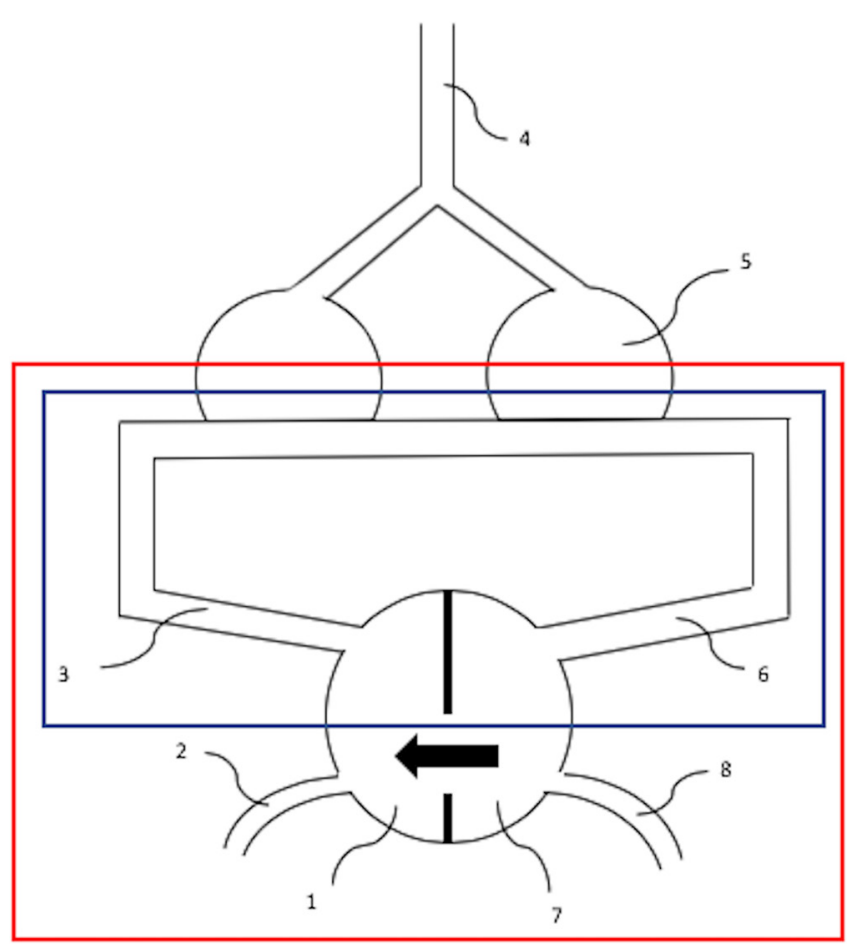

Figure 2 Illustration of the thoracic cardiovascular circulation in Atrial septal defect (arrow). The blue frame represents the pulmonary circulation measured with the IGR (Innocor®) and the red frame the systemic circulation measured with the TB (Physioflow $\AA$ ). 1. Right side of the heart; 2. Vena cava; 3. Pulmonary artery; 4. Trachea; 5. Lung; 6. Pulmonary Veins; 7 . Left side of the heart; 8 . Aorta.

$\left.\left(\mathrm{O}_{2}\right)\right) \cdot \mathrm{SF}_{6}$ is an insoluble inert gas which is used as a marker of gas mixture distribution homogeneity. $\mathrm{N}_{2} \mathrm{O}$ is a blood soluble gas which serves as gas tracer. Its disappearance from the lung is proportional to the rate of pulmonary blood flow. ${ }^{19} 20$ Rebreathing procedures will be achieved at a breathing rate of $20 / \mathrm{min}$, each separated by 3 min. Each rebreathing manoeuvre will last 15-20 s which represents a total of 25-30 cardiac cycles and will result in a cardiac output value. The average of the three cardiac output measurements will be used as mean for statistical analyses.

Under normal physiological conditions, Qp is equivalent to $\dot{\mathrm{Q} s}$. The absolute difference between $\dot{\mathrm{Qs}}_{\mathrm{TB}}$ and QpigR quantifies the intrathoracic shunt. The qualitative aspect of the intrathoracic shunt with a shunt fraction ratio such that $\dot{Q p} / \dot{Q}$ s ratio $>1.0$ reflects a left-to-right shunt. The severity of the shunt is expressed by the value of the $\dot{Q p} / \dot{Q}$ s ratio, such that higher shunt ratios reflect greater severity.

\section{Study outcomes}

Primary outcome

The main outcome of the study is the difference between Qp/Qs ratio measured by the double non-invasive technique ( $\dot{Q p}_{\mathrm{IGR}} / \mathrm{Qs}_{\mathrm{TB}}$ ) when compared with the Doppler echocardiography Qp/Qs ratio taken before interventional closure of secundum ASD. The working hypothesis is that there will be no significant difference between these ratios.

\section{Secondary outcomes}

The secondary outcomes are:

The Qp/Qs ratio measured by the double non-invasive

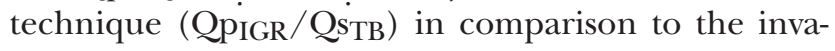
sive direct Fick method.

The $\dot{\mathrm{Qp}} / \dot{\mathrm{Q}}$ ratio measurements obtained after ASD closure by the double non-invasive technique (QpigR $/ \dot{\mathrm{Qs}}_{\mathrm{TB}}$ ) to those measured using Doppler echocardiography (Qp/QsieD2).

Relationship between extent of shunt QpigR/Qsi assessed by Fick, echocardiography and double approach and cardiac output measurements.

\section{Study limitations}

The study presents some methodological limitations:

First, there is a lack of control on patient rebreathing manoeuvre with the IGR. Second, some patient's factors such as preload or anxiety might influence physiological left-to-right shunt volumes before, during or after catheterisation to varying degrees. This bias becomes irrelevant after shunt closure, if shunt closure is completed. Third, the difference of time periods of data acquisition between the methods of cardiac output measurement (lower number of heart cycles captured by echocardiography, as compared with the other monitoring methods averaging more heart and respiratory cycles) might introduce a bias in shunt determination.

\section{Statistical considerations \\ Estimation of sample size}

To evaluate the agreement between the double non-invasive approach and the gold standard technique, a sample size of 30 subjects would provide a power of $90 \%$ to highlight an agreement of 0.8 (Lin's concordance coefficient) for a two-sided type I error at 5\%. A sequential exploratory analysis will be proposed each 10 patients without correction of type I error.

\section{Statistical analysis}

All statistical analyses will be performed using Stata software (V.13, StataCorp, College Station, USA). A two-sided type I error will be set at 0.05 to indicate statistical significance. Continuous data will be presented as mean $\pm \mathrm{SD}$ or as median (IQR), according to the statistical distribution. The assumption of normality will be studied with the Shapiro-Wilk test. The primary analysis will be performed using correlation coefficient (Pearson or Spearman according to the statistical distribution), Lin's concordance coefficient and Bland and Altman plot in order to study the accuracy between non-invasive Qp/Qs measurement and Doppler echocardiography in adults with ASD. The correlation and concordance coefficients will be presented with $95 \%$ CIs. 


\section{Secondary analysis}

In addition, the $\dot{Q p} / \dot{Q}$ s obtained using each method will be examined regardless of the size of ASD using correlation coefficient and analysis of variance (ANOVA) or Kruskal-Wallis test (if the assumptions of ANOVA are not met: normality and homoscedasticity studied by Bartlett test). The same statistical analyses will be carried out with respect to direct Fick measured shunt ratios. Finally, the intraindividual and interindividual reproducibility of the double non-invasive approach will be evaluated by intraclass correlation coefficient (ICC estimated from a mixed model with patient as random effect). ICC will be presented with a $95 \%$ CI.

\section{ETHICS AND DISSEMINATION Approval}

According to the French regulation on clinical trials, the study has been submitted to the 'Comité de Protection des Personnes Ouest V' (Reference 2017-A03149-44) and to the 'Agence Nationale de Sécurité du Medicament (ANSM)' (the French regulatory authority for research). Approval from the Ethics Review Board is dated 13 February 2018 and from the ANSM 30 January 2018. Any modification in the protocol or informed consent during the study will be presented to the reference authority. The study is currently registered on the clinical website under the number NCT03437148.

\section{Patient informed consent}

All patients will receive verbal and written information on the aim of the study and the protocol. Written informed consent will be obtained prior to their participation in the study. During the study, the patients will have the possibility to ask all questions concerning the protocol from the cardiologist or investigator. They will be informed that they are free to stop the study at any time at their own discretion.

\section{Data collection and quality management}

Data will be collected by the principal investigator and the trained clinical research assistant. Data will be registered in written notebooks at each assessment point for each patient. Data capture will be achieved using Research Electronic Data Capture. A clinical research assistant will be commissioned to ensure the progress of the study, and the data capture according to the standard operating procedures implemented at the University Hospital of Clermont-Ferrand in accordance with the Good Clinical Practice in current French laws.

\section{Access to data}

The data set will be the property of the institution. However, the principal investigator and the project manager will have full access to the final data set. The results will be communicated in a peer-reviewed journal, will be presented at an international conference and will appear in ClinicalTrials.gov.

\section{DISCUSSION}

To the best of our knowledge, our study is the first to assess the $\dot{Q}$ p/Qs ratio using a combined non-invasive approach of Qp/Qs ratio measurement using TB and IGR.

Cardiac output is a valuable physiological measurement to provide insight into integrated cardiocirculatory systemic functions at play to refine diagnosis and capability for several chronic disease states. ${ }^{18}$ To date, accurate measurement of cardiac output remains challenging in everyday clinical environments. Over the last decade, TB has been introduced which provides an interesting alternative as it allows to monitor values of continuous periods. ${ }^{10} 11$ This approach, however, may be limiting with respect to identifying right and left heart contributions. Rebreathing methodologies for cardiac output determinations provide such an opportunity. Peyton et al demonstrated the value of IGR to calculate the ratio $\mathrm{Qp} /$ Qs by combining the shunt equation and Fick principle. The limits of their approach stand in the impossibility to extend the application to intracardiac shunt. ${ }^{21}$

The aim of this study is to demonstrate the non-inferiority of the combined non-invasive approach of $\dot{\mathrm{Qp}} / \dot{\mathrm{Qs}}$ ratio measurement using TB and IGR in adults with secundum ASD by comparing the results obtained with those from usual methods (Doppler echocardiography and direct Fick). In this context, Perrault et al used TB and IGR simultaneously to measure the cardiac output either by TB, IGR or $\mathrm{CO}_{2}$ rebreathing (with or without correction by $\mathrm{PaCO}_{2}$ ) in patients with chronic obstructive pulmonary disease at rest and exercise. The ability of TB and IGR to correctively measure Qs and Qp, respectively, was confirmed. Interesting results lie on the possibility to estimate the shunt effect due to underestimation of cardiac output by IGR in comparison to TB.

The added value of the double non-invasive approach lies in the possibility to quantify and qualify the intrathoracic shunt from physiological haemodynamic parameters with relatively minor discomfort to the patient. The simultaneous use of IGR and TB is non-operator dependent, totally non-invasive and easy to use contrary to the Doppler echocardiography or the Fick methodology. In addition, the combined technique can be successfully used during clinical cardiopulmonary exercise testing enabling early or refined diagnoses or for follow-up and clinical management. ${ }^{522} 23$ Such an approach enables to quantify or qualify the shunt on exercise or shunt reversal and cyanosis which may first appear under exercise. ${ }^{24}$

Thus, the first step of this process is to evaluate the concomitant use of TB and IGR at rest in intracardiac shunt in comparison to standard methods.

\section{Author affiliations}

${ }^{1}$ Thoracic and Endocrine Surgery, Centre Jean Perrin, Clermont-Ferrand, France ${ }^{2}$ Cardiology and Vascular Department, Hopital Gabriel Montpied, Clermont-Ferrand, France

${ }^{3}$ Faculty of Health Sciences, University of Ottawa, Ottawa, Ontario, Canada ${ }^{4}$ Respiratory and Epidemiology Clinical Research Unit, Montreal Chest Institute, Montreal, Quebec, Canada

${ }^{5}$ Biostatistics Unit, Hopital Gabriel Montpied, Clermont-Ferrand, France 
${ }^{6}$ Department of Physiology and Medical Sport, Hopital Gabriel Montpied, ClermontFerrand, France

${ }^{7}$ INRA, UMR 1018, UNH, Université d'Auvergne, Centre de Recherche en Nutrition Humaine Auvergne, Clermont-Ferrand, France

Acknowledgements We sincerely thank the McGill University for the loan of the Innocor device.

Contributors LF, RR, AC, CD, JRL, BP, FC, HP and RT designed the study. LF, RR, AC, $\mathrm{CD}$ and $\mathrm{HP}$ read and corrected the drafts. $\mathrm{LF}, \mathrm{RR}, \mathrm{CD}$ and $\mathrm{AC}$ collected and managed the data. $L F, R R, C D, A C, J R L$ and $B P$ interpreted the data.

Funding The authors have not declared a specific grant for this research from any funding agency in the public, commercial or not-for-profit sectors.

Competing interests None declared.

Patient consent for publication Obtained.

Ethics approval The study protocol was approved by the French regulatory authority for research (Agence Nationale de Sécurité du Medicament et des Produits de Santé) and the Research Ethics Committee/Institutional Review Board (REC/IRB: Comité de Protection des Personnes Ouest V France) under number 2017-A03149-44.

Provenance and peer review Not commissioned; externally peer reviewed.

Open access This is an open access article distributed in accordance with the Creative Commons Attribution Non Commercial (CC BY-NC 4.0) license, which permits others to distribute, remix, adapt, build upon this work non-commercially, and license their derivative works on different terms, provided the original work is properly cited, appropriate credit is given, any changes made indicated, and the use is non-commercial. See: http://creativecommons.org/licenses/by-nc/4.0/.

\section{REFERENCES}

1. Perrault H, Richard R, Kapchinsky S, et al. Addressing Assumptions for the Use of Non-invasive Cardiac Output Measurement Techniques During Exercise in COPD. COPD 2016;13:75-81.

2. Geva T, Martins JD, Wald RM. Atrial septal defects. Lancet 2014;383:1921-32

3. Baumgartner H, Bonhoeffer P, De Groot NMS, et al. ESC Guidelines for the management of grown-up congenital heart disease (new version 2010). Eur Heart J 2010;31:2915-57.

4. Maatouk F, Ben Farhat M, Betbout F, et al. [Right ventricular dilatation and intraventricular septal motion after surgical closure of atrial septal defect]. Arch Mal Coeur Vaiss 2001;94:204-10.

5. Baumgartner H, Bonhoeffer P, De Groot NM, et al. ESC Guidelines for the management of grown-up congenital heart disease (new version 2010). Eur Heart J 2010;31:2915-57.

6. Dittmann $\mathrm{H}$, Voelker W, Karsch KR, et al. Influence of sampling site and flow area on cardiac output measurements by Doppler echocardiography. J Am Coll Cardiol 1987;10:818-23.

7. Silvestry FE, Cohen MS, Armsby LB, et al. Guidelines for the Echocardiographic Assessment of Atrial Septal Defect and Patent Foramen Ovale: From the American Society of Echocardiography and Society for Cardiac Angiography and Interventions. J Am Soc Echocardiogr 2015;28:910-58.

8. Quiñones MA, Otto CM, Stoddard M, et al. Recommendations for quantification of Doppler echocardiography: a report from the Doppler Quantification Task Force of the Nomenclature and Standards Committee of the American Society of Echocardiography. J Am Soc Echocardiogr 2002;15:167-84.

9. Miller HC, Brown DJ, Miller GA. Comparison of formulae used to estimate oxygen saturation of mixed venous blood from caval samples. Br Heart J 1974;36:446-51.

10. Charloux A, Lonsdorfer-Wolf E, Richard R, et al. A new impedance cardiograph device for the non-invasive evaluation of cardiac output at rest and during exercise: comparison with the "direct" Fick method. Eur J Appl Physiol 2000;82:313-20.

11. Richard R, Lonsdorfer-Wolf E, Charloux A, et al. Non-invasive cardiac output evaluation during a maximal progressive exercise test, using a new impedance cardiograph device. Eur J Appl Physiol 2001;85:202-7.

12. Miles DS, Gotshall RW, Golden JC, et al. Accuracy of electrical impedance cardiography for measuring cardiac output in children with congenital heart defects. Am J Cardiol 1988;61:612-6.

13. Braden DS, Leatherbury L, Treiber FA, et al. Noninvasive assessment of cardiac output in children using impedance cardiography. Am Heart J 1990;120:1166-72.

14. Peyton PJ, Chong SW. Minimally invasive measurement of cardiac output during surgery and critical care: a meta-analysis of accuracy and precision. Anesthesiology 2010;113:1220-35.

15. Fontana P, Boutellier U, Toigo M. Reliability of measurements with Innocor during exercise. Int J Sports Med 2009;30:747-53.

16. Dong $L$, Wang JA, Jiang CY. Validation of the use of foreign gas rebreathing method for non-invasive determination of cardiac output in heart disease patients. J Zhejiang Univ Sci B 2005;6:1157-62.

17. Gabrielsen A, Videbaek R, Schou M, et al. Non-invasive measurement of cardiac output in heart failure patients using a new foreign gas rebreathing technique. Clin Sci 2002;102:247-52.

18. Agostoni P, Cattadori G, Apostolo A, et al. Noninvasive measurement of cardiac output during exercise by inert gas rebreathing technique: a new tool for heart failure evaluation. J Am Coll Cardiol 2005;46:1779-81.

19. Damgaard M, Norsk $P$. Effects of ventilation on cardiac output determined by inert gas rebreathing. Clin Physiol Funct Imaging 2005;25:142-7.

20. Clemensen P, Christensen P, Norsk P, et al. A modified photo- and magnetoacoustic multigas analyzer applied in gas exchange measurements. J Appl Physiol 1994;76:2832-9.

21. Peyton PJ, Robinson GJ, McCall PR, et al. Noninvasive measurement of intrapulmonary shunting. J Cardiothorac Vasc Anesth 2004;18:47-52.

22. Van De Bruaene A, Buys R, Vanhees L, et al. Cardiopulmonary exercise testing and SF-36 in patients with atrial septal defect type secundum. J Cardiopulm Rehabil Prev 2011;31:308-15.

23. Komar M, Przewlocki T, Olszowska M, et al. The benefit of atrial septal defect closure in elderly patients. Clin Interv Aging 2014;9:1101-7.

24. Barron AJ, Wensel R, Francis DP, et al. The role for cardiopulmonary exercise testing in patients with atrial septal defects: a review. Int J Cardiol 2012;161:68-72. 